\title{
Restriction map in a regular reduction of $\mathrm{SU}(n)^{2 g}$
}

\author{
Sébastien Racanière
}

\begin{abstract}
The quasi-Hamiltonian reduction of $\mathbf{S U}(n)^{2 g}$ at a regular value, in the centre of $\mathbf{S U}(n)$, of the moment map is isomorphic to a moduli-space of semi-stable vector bundles over a Riemann surface. We describe the restriction map from the equivariant cohomology of $\mathbf{S U}(n)^{2 g}$ to the cohomology of the moduli space in terms of natural multiplicative generators of these cohomologies.
\end{abstract}

Mathematics Subject Classification (2000). 53D20, 14H60.

Keywords. Moduli spaces, symplectic reduction, quasi-Hamiltonian spaces, SU $(n)$.

\section{Motivations}

All cohomologies will be taken with coefficients in the field $\mathbf{Q}$ of rational numbers. For a compact connected Lie group $G$, we denote $E G \longrightarrow B G$ the universal principal $G$-bundle. If $G$ acts on a manifold $M$, we denote $(M)_{G}$ the space $M \times_{G}$ $E G$. The equivariant cohomology $H_{G}^{*}(M)$ of $M$ with respect to the action of $G$ is by definition the Cech cohomology of $(M)_{G}$. For an account of equivariant cohomology see [6] and [14].

Let $g$ be an integer bigger than 1 . Let $\pi$ be the group

$$
\pi=\left\langle a_{1}, b_{1}, \ldots, a_{g}, b_{g}, c ; \prod_{k=1}^{g}\left[a_{k}, b_{k}\right]=c, c^{n}=1\right\rangle .
$$

Let $n$ and $d$ be integers with $n$ bigger than 1 and let $\zeta$ be the $n$-th root of unity $\zeta=e^{-2 \pi i \frac{d}{n}}$. Put $\beta=\zeta \mathbf{I}$, where $\mathbf{I}$ is the identity matrix in the special unitary group $\mathbf{S U}(n)$. We define

$$
S_{\beta}=\{\rho \in \operatorname{Hom}(\pi, \mathbf{S U}(n)) \mid \rho(c)=\beta\}
$$

the space of $\mathbf{S U}(n)$-representations of $\pi$ such that $\beta$ is the image of $c$. Because $\beta$ is in the centre of $\mathbf{S U}(n)$, the group $\mathbf{S U}(n)$ acts on $S_{\beta}$ and its quotient

$$
\mathfrak{m}_{\beta}=S_{\beta} / \mathbf{S U}(n)
$$

is the moduli space of $\mathbf{S U}(n)$-representations of $\pi$ that send $c$ to $\beta$. 
Narasimhan and Seshadri [17] have shown that $\mathfrak{m}_{\beta}$ is isomorphic to the moduli space of holomorphic semi-stable vector bundles of rank $n$, degree $d$, and fixed determinant over a compact Riemann surface $X$ of genus $g$. For $d$ and $n$ co prime, $\mathfrak{m}_{\beta}$ is compact and smooth. In this case, Atiyah and Bott [1] showed that this space is symplectic, proposed a family of multiplicative generators of its cohomology and gave an inductive formula (on the rank $n$ ) for the Betti numbers of $\mathfrak{m}_{\beta}$. Their method consists in studying an infinite dimensional Hamiltonian space. In 1993, Huebschmann [8] and Jeffrey [10] independently gave a group cohomology construction of the symplectic form on $\mathfrak{m}_{\beta}$ (their results are summarised in a joint paper [9]). In 1998, Alekseev, Malkin and Meinrenken [2] showed that Huebschmann and Jeffrey's construction fits in a more general setting: one can get the moduli space $\mathfrak{m}_{\beta}$ (and many others, moduli spaces of flat connections on a principal bundle) as the Marsden-Weinstein reduction of a quasi-Hamiltonian space. This space is $\mathbf{S U}(n)^{2 g}$, it is relatively simple in contrast with the usual descriptions of $\mathfrak{m}_{\beta}$ as a Hamiltonian reduction. A quasi-Hamiltonian (or q-Hamiltonian for short) space is a Hamiltonian space with a group valued moment map. Its 2 -form is not symplectic in general but the Marsden-Weinstein reduction is well defined and the reduced space is symplectic.

An important result about Hamiltonian spaces is the

Theorem 0.1 (Kirwan). Let $M$ be a symplectic manifold. Assume $G$ is a compact Lie group acting symplectically on $M$ and assume there exists a moment map $\phi$ for this action. Let 0 be the null vector of the dual of the Lie algebra of $G$. The restriction map

$$
H_{G}^{*}(M) \longrightarrow H_{G}^{*}\left(\phi^{-1}(0)\right)
$$

is surjective.

It is a natural question to ask if this theorem is still true for q-Hamiltonian spaces. It is quite easy to see that the answer is no. For example, to get $\mathfrak{m}_{\beta}$, one considers $\mathbf{S U}(n)^{2 g}$ with moment map $\mu$

$$
\begin{gathered}
\mathbf{S U}(n)^{2 g} \\
\left(A_{1}, B_{1}, \ldots, A_{g}, B_{g}\right) \longmapsto \\
\prod_{k=1}^{g}\left[A_{k}, B_{k}\right]
\end{gathered}
$$

the product of the commutators and a certain 2-form (see [2] for more details). Then the reduced space at $\beta$ being symplectic and compact, its degree two cohomology (which is isomorphic to $H_{\mathbf{S U}(n)}^{2}\left(\mu^{-1}(\beta)\right)$ ) contains a non trivial class whereas $H_{\mathbf{S U}(n)}^{2}\left(\mathbf{S U}(n)^{2 g}\right)=\{0\}$. Thus the map

$$
r: H_{\mathbf{S U}(n)}^{*}\left(\mathbf{S U}(n)^{2 g}\right) \longrightarrow H_{\mathbf{S U}(n)}^{*}\left(\mu^{-1}(\beta)\right)
$$

is not surjective.

Our aim is to give a description of this last map $r$ (Theorem 5.1) when $d$ and $n$ are co prime. Note that in [3], a theorem of localisation in the context of quasi- 
Hamiltonian spaces is given. It may be interesting to see how our theorem could be used to apply this localisation theorem to the reduction of $\mathbf{S U}(n)^{2 g}$ at $\beta$.

This paper is organised in the following way. Section 2 gives a (very short) review of the prerequisites on q-Hamiltonian spaces and semi-stable bundles. In particular, Narasimhan and Seshadri's theorem (see Theorems 2.9 and 2.13) is used throughout this article to identify $\mathfrak{m}_{\beta}$ with $\mu^{-1}(\beta) / \mathbf{S U}(n)$ and $H^{*}\left(\mathfrak{m}_{\beta}\right)$ with $H_{\mathbf{S U}(n)}^{*}\left(\mu^{-1}(\beta)\right)$. In Section 3 , we give a construction of a universal bundle on $\mathfrak{m}_{\beta} \times X$, we then recall how Biswas and Raghavendra [4] use this bundle to define a set $\left\{a_{k}, b_{k, j}, d_{k}, 2 \leq k \leq n, 1 \leq j \leq 2 g\right\}$ of canonical multiplicative generators of the cohomology of $\mathfrak{m}_{\beta}$ (Theorem 3.4). In the next section we define a bundle on $\mathbf{S U}(n)^{2 g} \times X-\{$ point $\}$ and use it to get a set $\left\{c_{k}, \sigma_{k, j}, 2 \leq k \leq n, 1 \leq j \leq g\right\}$ of multiplicative generators for the equivariant cohomology of $\mathbf{S U}(n)^{2 g}$ (Theorems 4.4 and 4.6). Finally in Section 5 we prove the

Theorem 5.1. The restriction map

$$
r: H_{\mathbf{S U}(n)}^{*}\left(\mathbf{S U}(n)^{2 g}\right) \longrightarrow H_{\mathbf{S U}(n)}^{*}\left(\mu^{-1}(\zeta \mathbf{I})\right)
$$

is given by

$$
\begin{aligned}
r\left(c_{k}\right) & =a_{k} \text { for } k=2, \ldots, n \\
r\left(\sigma_{k, j}\right) & =b_{k, j} \text { for } k=2, \ldots, n, j=1, \ldots, 2 g .
\end{aligned}
$$

\section{Acknowledgement}

I would like to thank M. Audin and F. Kirwan for their help in writing this article. I would also like to thank J. Huebschmann and L. Jeffrey for sending their articles to me, they have been very useful. Finally, I would like to thank the referee for his reading and his comments.

\section{Prerequisites}

In paragraph 2.1, we recall the definition of semi-stability for holomorphic vector bundles and state Narasimhan and Seshadri's theorem (Theorem 2.9). Then in $\S 2.2$, we give the definition of a q-Hamiltonian space and restate Narasimhan and Seshadri's result in the language of q-Hamiltonian spaces (Theorem 2.13).

\subsection{Semi-stable bundle}

The following constructions are due to Narasimhan and Seshadri [17]. Apart from the proof of Proposition 2.1, everything in this paragraph is from their article. 
Let $X$ be a Riemann surface of genus $g, g \geq 2$. Fix a point $x_{0}$ of $X$. We will first give a construction of a ramified covering $Y \longrightarrow X$ used in [17].

Proposition 2.1. There exists a simply connected covering

$$
p: Y \longrightarrow X
$$

with only one point of ramification $x_{0}$ of order $n$. Outside of this point, the map

$$
Y-\left\{p^{-1}\left(x_{0}\right)\right\} \longrightarrow X-\left\{x_{0}\right\}
$$

is a covering with group

$$
\pi=\left\langle a_{1}, b_{1}, \ldots, a_{g}, b_{g}, c ; c=\prod_{k=1}^{g}\left[a_{k}, b_{k}\right], c^{n}=1\right\rangle .
$$

Proof. We start by constructing $Y$, then we show that it is simply connected. Let $D$ be an open neighbourhood of $x_{0}$ biholomorphic to an open disc in $\mathbf{C}$ centred at zero. Let $D^{\prime}=D-\left\{x_{0}\right\}$. The fundamental group of $X^{\prime}=X-\left\{x_{0}\right\}$ has a presentation

$$
\pi_{1}\left(X^{\prime}\right)=\left\langle a_{1}, b_{1}, \ldots, a_{g}, b_{g}\right\rangle,
$$

such that the element $\prod_{k=1}^{g}\left[a_{k}, b_{k}\right]$ is the class of a small circle $\gamma$ included in $D^{\prime}$ and going counter clockwise around $x_{0}$. Let $\pi$ be the group

$$
\pi=\left\langle a_{1}, b_{1}, \ldots, a_{g}, b_{g}, c ; c=\prod_{k=1}^{g}\left[a_{k}, b_{k}\right], c^{n}=1\right\rangle .
$$

The natural surjective map

$$
\pi_{1}\left(X^{\prime}\right) \longrightarrow \pi
$$

defines a galoisian covering

$$
p: Y^{\prime} \longrightarrow X^{\prime}
$$

with group $\pi$. Fix a point $x_{1}$ of $D^{\prime}$. We take it as the base point for the fundamental groups of $X, X^{\prime}$ and $D^{\prime}$. Let us decompose $p^{-1}\left(D^{\prime}\right)$ in its different connected components

$$
p^{-1}\left(D^{\prime}\right)=\bigcup_{\alpha \in \Lambda} V_{\alpha}
$$

Each

$$
V_{\alpha} \stackrel{p}{\longrightarrow} D^{\prime}
$$

is a connected covering. As $D^{\prime}$ is a disc minus its centre, this last covering group is generated by the element in $\pi$ corresponding to the loop $\gamma$. The connected covering of a disc with its centre removed is either the upper half complex plane with the exponential as a projective map or a disc with its centre removed and 
a projection map of the type $z \longmapsto z^{m}$, where $m$ is a positive integer. Here, the class of $\gamma$ acts as $c$, thus $V_{\alpha}$ is a disc with its centre removed and, for each $\alpha$,

$$
V_{\alpha} \stackrel{p}{\longrightarrow} D^{\prime}
$$

is the map $z \longmapsto z^{n}$. Let

$$
Y=\left(Y^{\prime} \bigcup_{\alpha \in \Lambda}(D, \alpha)\right) / \sim
$$

where for $y \in Y^{\prime}$ and $(x, \alpha) \in(D, \alpha)$

$$
y \sim(x, \alpha) \text { if and only if } p(x)=y .
$$

The natural projection

$$
Y \longrightarrow X
$$

is a covering with a unique ramification point at $x_{0}$ with order $n$. We now have to check that $Y$ is simply connected. We get $Y^{\prime}$ from $Y$ by removing a discrete set of points. Hence the map

$$
\pi_{1}\left(Y^{\prime}\right) \longrightarrow \pi_{1}(Y)
$$

is surjective. The sequence

$$
\{1\} \longrightarrow \pi_{1}\left(Y^{\prime}\right) \longrightarrow \pi_{1}\left(X^{\prime}\right) \longrightarrow \pi \longrightarrow\{1\}
$$

is exact. The kernel of $\pi_{1}\left(X^{\prime}\right) \longrightarrow \pi$ is the normal subgroup generated by $c^{n}$. Let $a$ in $\pi_{1}\left(X^{\prime}\right)$ be the class of a loop $\eta:[0,1] \longrightarrow X^{\prime}$. The class of $\gamma^{n}$ is $c^{n}$. Let us lift $\eta \cdot \gamma^{n} \cdot \eta^{-1}$ in $Y^{\prime}$. To do so we have to take a lift $\tilde{\eta}$ of $\eta$ in $Y^{\prime}$ and then take a lift $\widetilde{\gamma}^{n}$ of $\gamma^{n}$ satisfying $\widetilde{\gamma}^{n}(0)=\widetilde{\eta}(1)$. The loop we wanted is $\widetilde{\eta} \cdot \widetilde{\gamma}^{n} \cdot \widetilde{\eta}^{-1}$. There exists $\alpha$ such that

$$
\widetilde{\gamma}^{n} \subset V_{\alpha}
$$

thus $\widetilde{\gamma}^{n}$ is homotopic to the constant loop in $Y$. The image of $a \cdot c^{n} \cdot a^{-1}$ by $\pi_{1}\left(Y^{\prime}\right) \longrightarrow \pi_{1}(Y)$ is 1 , hence the image of $\pi_{1}\left(Y^{\prime}\right) \longrightarrow \pi_{1}(Y)$ is $\{1\}$ and

$$
\pi_{1}(Y)=\{1\} .
$$

Choose a $y_{0}$ in $p^{-1}\left(x_{0}\right)$. In the presentation

$$
\pi=\left\langle a_{1}, b_{1}, \ldots, a_{g}, b_{g}, c ; \prod_{k=1}^{g}\left[a_{k}, b_{k}\right]=c, c^{n}=1\right\rangle
$$

of $\pi$, we can assume that $c$ is a generator of the isotropy group $\pi_{y_{0}}$ of $y_{0}$.

For a representation $\rho: \pi \rightarrow \mathbf{G L}(n)$ of $\pi$, we denote $E_{\pi}(\rho)$ the vector bundle

$$
Y \times \mathbf{C}^{n} \longrightarrow Y
$$

with the action:

$$
\begin{gathered}
\pi \times\left(Y \times \mathbf{C}^{n}\right) \\
(\gamma,(y, v))
\end{gathered} \begin{gathered}
Y \\
\longmapsto(\gamma \cdot y, \rho(\gamma) v) .
\end{gathered}
$$


Let $\mathbf{E}$ be the sheaf of germs of holomorphic sections of $E_{\pi}(\rho)$. The group $\pi$ acts on the image sheaf $p_{*}(\mathbf{E})$. Let $p_{*}^{\pi}(\mathbf{E})$ be the subsheaf of $\pi$-invariant elements of $p_{*}(\mathbf{E})$. It is a rank $n$ locally free sheaf of $\mathbf{O}_{\mathbf{X}}$-modules. It defines a holomorphic vector bundle, $p_{*}^{\pi}\left(E_{\rho}\right)$, of rank $n$ on $X$. A set of transition functions is obtained in the following way. Let $\left\{U_{i}\right\}_{i=0}^{m}$ be a finite open covering of $X$ satisfying:

(1) all non-empty intersections of sets of the type $U_{i}$ is contractible,

(2) $x_{0} \in U_{0}$ and $\bigcup_{i=1}^{m} U_{i}=X-\left\{x_{0}\right\}$,

(3) there exist discs $\left\{D_{i}\right\}_{i=0}^{m}$ in $Y$ such that $y_{0} \in D_{0}$ and $U_{0}$ is the quotient of $D_{0}$ by $\pi_{y_{0}}$, the restriction $\left.p\right|_{D_{i}}$ is an homeomorphism of $D_{i}$ with $U_{i}$, for all non zero $i$.

For each triplet $i, j, k$, choose a connected component $W_{i j, k}$ of $p^{-1}\left(U_{i} \cap U_{j}\right) \cap D_{k}$. If $U_{i} \cap U_{j}$ is not empty, we denote $\gamma_{i, j}$ the element of $\pi$ satisfying $\gamma_{i, j} W_{i j, j}=W_{j i, i}$. According to $[17$, p. 550] :

Proposition 2.2. On each $\left\{U_{i}\right\}_{i=0}^{m}$, the bundle $p_{*}^{\pi}\left(E_{\rho}\right)$ is trivial and a set of transition functions is given by:

$$
\begin{cases}g_{i, j}=\rho\left(\gamma_{i, j}\right) & \text { in } U_{i} \cap U_{j}, \text { for } i, j \neq 0 \\ g_{0, i}=f_{0, i} \rho\left(\gamma_{0, i}\right) & \text { in } U_{0} \cap U_{i}, \text { for } i \neq 0\end{cases}
$$

where $f_{0, i}: U_{0} \cap U_{i} \rightarrow \mathbf{C}^{*}$ depends only on $\tau$.

Definition 2.3. Let $W$ be a degree $d(W)$ and rank $r(W)$ holomorphic vector bundle on $X$. It is said to be stable, resp. semi-stable, if for each proper subbundle $V$, we have

$$
\frac{d(V)}{r(V)}<\frac{d(W)}{r(W)}, \text { resp. } \frac{d(V)}{r(V)} \leq \frac{d(W)}{r(W)} .
$$

Remark 2.4. If $d(W)$ and $r(W)$ are co prime then the notions of stability and semi-stability are equivalent.

Recall that $d$ is an integer, $0 \leq d \leq n-1$, and $\zeta=e^{-2 \pi i \frac{d}{n}}$ is an $n$-th root of unity. Let $z$ be a coordinate in a neighbourhood of $y_{0}$ such that $\pi_{y_{0}}$ is the group of multiplications by $\zeta^{k}$. Up to a change of generator $c$ of $\pi_{y_{0}}$, we can assume that $c$ acts by multiplication by $e^{\frac{2 i \pi}{n}}$. Let $\tau$ be the character of $\pi_{y_{0}}$ defined by $\tau(c)=\zeta$. A representation $\rho: \pi \rightarrow \mathbf{U}(n)$ is said to be of type $\tau$ if for all $\gamma \in \pi_{y_{0}}$, we have $\rho(\gamma)=\tau(\gamma)$ I. For any representation $\rho$ of type $\tau$ we have:

$$
d\left(p_{*}^{\pi}\left(E_{\rho}\right)\right)=d-n \quad(\text { see }[5, \text { p. } 13]) .
$$

Again, according to [17]:

Theorem 2.5. A holomorphic vector bundle of rank $n$ and degree $d-n$ on $X$ is semi-stable if and only if it is isomorphic to a $p_{*}^{\pi}\left(E_{\rho}\right)$, where $\rho: \pi \rightarrow \mathbf{U}(n)$ is a unitary representation of type $\tau$. This bundle is stable if and only if the 
representation $\rho$ is irreducible. Moreover, two such bundles are isomorphic if and only if their corresponding unitary representations are isomorphic.

Remark 2.6. For $d$ and $n$ co prime, any representation $\rho: \pi \rightarrow \mathbf{U}(n)$ of type $\tau$ is irreducible [17, Prop. 9.3].

Let $\mathfrak{n}$ be the moduli space of rank $n$, degree $d$, stable holomorphic vector bundles over $X$.

Remark 2.7. Let $M$ be a holomorphic line bundle of degree 1 over $X$ (it always exists). The moduli space of rank $n$ stable holomorphic vector bundles with fixed determinant of degree $d-n$ over $X$ is isomorphic to the moduli space of rank $n$ stable holomorphic vector bundles with fixed determinant of degree $d$ over $X$. The isomorphism is induced by the map which to a bundle $E \rightarrow X$ associates $E \otimes M$.

We fix such a bundle $M$ and use it to identify the two moduli spaces of Remark 2.7. Thus we have

Theorem 2.8. The moduli space $\mathfrak{n}$ is isomorphic to the quotient of the space of unitary representations of type $\tau$ of $\pi$ by the action of $\mathbf{U}(n)$.

The map which to a class of bundles in $\mathfrak{n}$ associates its determinant is a fibration over the moduli space of line bundles of degree $d$. Its fibre is called the moduli space of rank $n$ stable holomorphic line bundles over $X$ with fixed determinant (of degree $d$ ). We get all such bundles by taking only representations $\rho: \pi \rightarrow$ $\mathbf{S U}(n)$ of type $\tau$. Let $S$ be the set of such representations. We identify it with $\left\{\left(A_{1}, B_{1}, \ldots, A_{g}, B_{g}\right) \in \mathbf{S U}(n)^{2 g} \mid \prod_{k=1}^{g}\left[A_{k}, B_{k}\right]=\zeta \mathbf{I}\right\}$ by:

$$
\begin{aligned}
& S \longrightarrow\left\{\left(A_{1}, B_{1}, \ldots, A_{g}, B_{g}\right) \in \mathbf{S U}(n)^{2 g} \mid \prod_{k=1}^{g}\left[A_{k}, B_{k}\right]=\zeta \mathbf{I}\right\} \\
& \rho \longmapsto\left(\rho\left(a_{1}\right), \rho\left(b_{1}\right), \ldots, \rho\left(a_{g}\right), \rho\left(b_{g}\right)\right) .
\end{aligned}
$$

The action of $\mathbf{S U}(n)$ on the representations becomes, under this identification, the diagonal action by conjugation of $\mathbf{S U}(n)$ on $\mathbf{S U}(n)^{2 g}$.In this article we work with $\mathfrak{m}$ rather than $\mathfrak{n}$. We have:

Theorem 2.9. Let $d$ be an integer, $1 \leq d \leq n-1$, co prime with $n$. Let $\mathfrak{m}$ be the moduli space of rank $n$ holomorphic stable vector bundles over $X$ with fixed determinant (of degree d). The map

$$
\begin{aligned}
& S \longrightarrow \mathfrak{m} \\
& \rho \longmapsto p_{*}^{\pi}\left(E_{\rho}\right)
\end{aligned}
$$

is a $\mathbf{P S U}(n)$-principal bundle. 
Proof. The only thing that is left to check is that for a representation $\rho=$ $\left(A_{1}, B_{1}, \ldots, A_{g}, B_{g}\right) \in S$ (recall we have identified $S$ with a set of matrices), its stabiliser $\operatorname{Stab}(\rho)$ is the centre of $\mathbf{S U}(n)$. Let $C$ be in the stabiliser of $\rho$. Let $\lambda$ be an eigenvalue of $C$ and let $E_{\lambda}$ be its eigenspace. As $C$ commutes with each of the $A_{i}, B_{i}$, the subspace $E_{\lambda}$ is stable by the unitary representation $\rho$. As $\rho$ is irreducible, $E_{\lambda}=\mathbf{C}^{n}$ and $C$ is in the centre of $\mathbf{S U}(n)$. On the other hand, any matrix in the centre of $\mathbf{S U}(n)$ does leave $\rho$ invariant. We have indeed a free action of $\operatorname{PSU}(n)$ on $S$.

\subsection{Quasi-Hamiltonian spaces}

The definition of a q-Hamiltonian space is due to Alekseev, Malkin and Meinrenken. Roughly speaking this is a Hamiltonian space with a group valued moment map. When the group is a torus, the definition reduces to the usual one of a Hamiltonian torus action whose moment map takes its values in the torus itself (see McDuff [15] and Weitsmann [19]).

Let $G$ be a compact Lie group. Let $\theta$ and $\bar{\theta}$ be respectively the left invariant and right invariant Maurer-Cartan forms on $G$. Choose a $G$-invariant scalar product $\langle$,$\rangle on the Lie algebra \mathfrak{g}$ of $G$. Define a 3 -form $\chi$ on $G$ by

$$
\chi=\frac{1}{12}\langle[\theta, \theta], \theta\rangle \text {. }
$$

Definition $2.10([2])$. Let $(M, G, \omega, \mu)$ be a 4-tuple where $M$ is a manifold acted on by a compact Lie group $G, \omega$ is a $G$-invariant 2 -form on $M$ and $\mu$ is an equivariant map from $M$ to $G$ (for the action by conjugation of $G$ on itself). This 4-tuple (or simply $M$ if there is no risk of confusion) is a q-Hamiltonian space if

(B1) $d \omega=-\mu^{*} \chi$

(B2) $\iota\left(v_{\xi}\right) \omega=\frac{1}{2} \mu^{*}\langle\theta+\bar{\theta}, \xi\rangle$

(B3) $\operatorname{ker} \omega_{x}=\left\{v_{\xi}(x) \mid \xi \in \operatorname{ker} \operatorname{Ad}_{\mu(x)}+1\right\}$.

The map $\mu$ is called the moment map.

This definition is a generalisation of the definition of a Hamiltonian space in the sense that any compact Hamiltonian space can be endowed with a q-Hamiltonian structure (this is an easy corollary of [2, Prop. 3.4.]).

A first example of a q-Hamiltonian space is a conjugacy class in a Lie group with moment map the inclusion of the conjugacy class in the group (see [2, §3]). The example that will be of interest to us is

Theorem 2.11 ([2]). Let $G$ be a compact Lie group and $g \geq 1$ an integer. There exists a 2 -form $\omega$ on $G^{2 g}$ such that the map

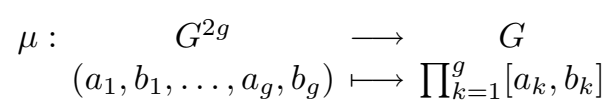


and the diagonal action of $G$ on $G^{2 g}$ by conjugation makes $\left(G^{2 g}, G, \omega, \mu\right)$ into a q-Hamiltonian space.

In particular we will apply this theorem with $G=\mathbf{S U}(n)$. An important fact about q-Hamiltonian spaces is that one can take their Marsden-Weinstein reduction. More precisely:

Theorem $2.12([2])$. Let $(M, G, \omega, \mu)$ be a q-Hamiltonian space. Let $h$ be in the centre of $G$. The moment map $\mu$ is a submersion at $x \in M$ if and only if the stabiliser of $x$ in $G$ is finite. If this is the case for any point of $\mu^{-1}(h)$, the reduced space $\mu^{-1}(h) / G$ is an orbifold (a manifold if the action of $G$ on $\mu^{-1}(h)$ is principal) on which the restriction of $\omega$ to $\mu^{-1}(h)$ descends to define a symplectic form. We call this space the reduction of $M$ at $h$.

As a corollary of Theorems 2.2, 2.12 and 2.9 we have:

Theorem 2.13. Let $n, d$ be co prime integers, $n \geq 2$ and $0 \leq d \leq n-1$. Let $\zeta=e^{-2 \pi i \frac{d}{n}}$ be an $n$-th root of unity and $\beta=\zeta \mathbf{I}$ in the centre of $\mathbf{S} \mathbf{U}(n)$. The moduli space $\mathfrak{m}_{\beta}$ of rank $n$ stable holomorphic vector bundles with fixed determinant (and degree d) over a Riemann surface $X$ of genus $g$ is isomorphic to the reduction of the $q$-Hamiltonian space $\mathbf{S U}(n)^{2 g}$ at $\beta$. It is a compact smooth symplectic manifold.

\subsection{Characteristic classes of principal bundles}

Following Biswas and Raghavendra [4], we define in this section some characteristic classes of a projective bundle. We will see that when the projective bundle comes from a vector bundle of degree 0, these characteristic classes are the same as the Chern classes of the vector bundle.

Let $\mathbf{Q}\left[X_{1}, \ldots, X_{n}\right]$ be a polynomial ring in $n$ variables. The cohomology of $B \mathbf{U}(n)$ is isomorphic to the subalgebra of invariant polynomials in the algebra $\mathbf{Q}\left[X_{1}, \ldots, X_{n}\right]$, under the action of the symetric group $S_{n}$ on the variables. For $k$ an integer in $[1, n]$, the Chern class $c_{k}$ in $H^{*}(B \mathbf{U}(n))$ corresponds to the Schur polynomial

$$
\sum_{1 \leq i_{1}<\cdots<i_{k} \leq n} X_{i_{1}} \ldots X_{i_{k}} .
$$

The projection from $\mathbf{U}(n)$ to $\mathbf{P} \mathbf{U}(n)$ defines a fibration $B \mathbf{U}(n) \longrightarrow B \mathbf{P U}(n)$ with fiber $B \mathbf{U}(1)$. This fibration is cohomologically trivial and $H^{*}(B \mathbf{P} \mathbf{U}(n))$ injects into $H^{*}(B \mathbf{U}(n))$. Let us define

$$
Y_{k}=X_{k}-\frac{1}{n} \sum_{k=1}^{n} X_{k} .
$$

The image of $H^{*}(B \mathbf{P} \mathbf{U}(n))$ in $H^{*}(B \mathbf{U}(n))$ is the ideal generated by the polyno- 
mials

$$
p_{k}=\sum_{1 \leq i_{1}<\cdots<i_{k} \leq n} Y_{i_{1}} \ldots Y_{i_{k}}, \text { for } 2 \leq k \leq n .
$$

The $k$-th characteristic class of a projective bundle over a manifold $M$ is the pullback of $p_{k}$ under the classifying map $M \longrightarrow B \mathbf{P U}(n)$.

For a vector bundle $F$ of degree 0 , that is when the first Chern class vanishes, we have $p_{k}(F)=c_{k}(F)$ for $k$ in $[2, n]$. It will be the case in particular if the structure group of the vector bundle is $\mathbf{S U}(n)$. This corresponds to the fact that the projection $B \mathbf{S U}(n) \longrightarrow B \mathbf{P U}(n)$ defines an isomorphism in cohomology.

\section{Construction of a universal bundle}

In this section, we fix $d, n, \zeta$ and $\beta$ as in Theorem 2.13. We use the notations of that theorem and of Theorem 2.2 with $G=\mathbf{S U}(n)$. We construct a universal bundle on $\mathfrak{m}_{\beta}$, that is a vector bundle $U$ over $\mathfrak{m}_{\beta} \times X$, holomorphic in the $X$ direction, such that for any class $[E]$ in $\mathfrak{m}_{\beta}$, the restriction of $U$ to $\{[E]\} \times X$ is in the class $[E]$. We then use this bundle to define natural multiplicative generators of the cohomology of $\mathfrak{m}_{\beta}$.

Recall that we defined page 399 an open covering of $X$ by subsets $\left\{U_{i}\right\}_{i=0}^{m}$. Define a complex vector bundle $T$ over $S \times X$ (where we have identified $S$ to $\left.\mu^{-1}(\zeta \mathbf{I})\right)$ as being trivial over the $S \times U_{i}$ and with transition functions:

$$
\begin{aligned}
&\left(S \times U_{i}\right) \cap\left(S \times U_{j}\right) \longrightarrow \begin{array}{c}
\mathbf{U}(n) \\
(\rho, x)
\end{array} \\
& \qquad\left\{\begin{array}{cl}
\rho\left(\gamma_{i, j}\right) & x \in U_{i} \cap U_{j}, i, j \neq 0 \\
f_{0, i}(x) \rho\left(\gamma_{0, i}\right) & x \in U_{0} \cap U_{i}, i \neq 0
\end{array} .\right.
\end{aligned}
$$

According to Proposition 2.2:

Proposition 3.1. The bundle $T$ satisfies: for all $\rho$ in $S$

$$
\left.T\right|_{\{\rho\} \times X} \cong p_{*}^{\pi}\left(E_{\rho}\right) .
$$

Define an action of $\mathbf{S U}(n)$ on $T$ by defining it on each $\left.T\right|_{S \times U_{i}}$ by

$$
\begin{aligned}
& \mathbf{S U}(n) \times\left(S \times U_{i} \times \mathbf{C}^{n}\right) \longrightarrow S \times U_{i} \times \mathbf{C}^{n} \\
& (g,(\rho, x, u)) \quad \longmapsto(g \cdot \rho, x, g(u)) .
\end{aligned}
$$

This action is well defined because if $x \in U_{j} \cap U_{i}$ and $t=(\rho, x, u)$ is in $S \times U_{i} \times \mathbf{C}^{n}$, then in the trivialisation $S \times U_{j} \times \mathbf{C}^{n}, t$ is written $t=\left(\rho, x, v(x) \rho\left(\gamma_{i, j}\right)(u)\right)$ where $v(x)$ is a scalar and

$$
\begin{aligned}
g \cdot\left(\rho, x, v(x) \rho\left(\gamma_{i, j}\right)(u)\right) & =\left(g \cdot \rho, x, g\left(v(x) \rho\left(\gamma_{i, j}\right)(u)\right)\right) \\
& =\left(g \cdot \rho, x, v(x) g \rho\left(\gamma_{i, j}\right) g^{-1} g(u)\right) .
\end{aligned}
$$

This last term is $(g \cdot \rho, x, g(u))$ written in $S \times U_{j} \times \mathbf{C}^{n}$. This action is a lift for the action of $\mathbf{S U}(n)$ on $S \times X$. Unfortunately it does not come from an action of 
$\operatorname{PSU}(n)$ and the bundle $T$ does not descend to a bundle on $\mathfrak{m} \times X$. Indeed the centre $\mathbf{Z} / n \mathbf{Z}$ of $\mathbf{S U}(n)$ acts trivially on $S$ but the generator $\zeta \mathbf{I}$ of $\mathbf{Z} / n \mathbf{Z}$ acts by multiplication by $\zeta$ in the fibres. To overcome this problem, we can construct a line bundle $L$ on $S$ with an action of $\mathbf{S U}(n)$ lifting the action on $S$ and such that $\zeta \mathbf{I}$ also acts by multiplication by $\zeta$ in the fibres. We will also denote $L$ the induced bundle on $S \times X$. The bundle $T \otimes L^{*}$ has the property of Proposition 3.1 but the action of $\mathbf{S U}(n)$ reduces to an action of $\mathbf{P S U}(n)$. By taking the quotient we get

Proposition 3.2. Let $M$ be the line bundle of Remark 2.7. The bundle

$$
U=M \otimes\left(T \otimes L^{*}\right) / \mathbf{P S U}(n) \longrightarrow \mathfrak{m} \times X
$$

is a universal bundle for $\mathfrak{m}_{\beta}$. That is, if $[E] \in \mathfrak{m}_{\beta}$ is the class of a bundle $E \rightarrow X$ then $\left.U\right|_{[E] \times X}$ is isomorphic to $E$.

We still have to prove the existence of the bundle $L$.

Lemma 3.3. There exists a line bundle $L$ over $S$ with an action of $\mathbf{S U}(n)$ lifting the one of $\mathbf{S U}(n)$ on $S$. This action satisfies: $\zeta \mathbf{I}$ acts by multiplication by $\zeta$ in the fibres.

Proof. The proof is inspired from [16].

The bundle $M \otimes T$ is a family (parameterised by $S$ ) of rank $n$, degree $d$ stable holomorphic vector bundles. Let $E$ be in this family and let $k$ be an integer. By Serre duality,

$$
H^{1}\left(E \otimes\left(\Omega_{X}^{1}\right)^{k}\right)=H^{0}\left(E^{\vee} \otimes\left(\Omega_{X}^{1}\right)^{1-k}\right)^{*}
$$

and this is the null vector space. Otherwise there would exist a non zero homomorphism $\left(\Omega_{X}^{1}\right)^{k-1} \rightarrow E^{\vee}$ and thus a subbundle of $E^{\vee}$ of degree bigger than or equal to $2(g-1)(k-1) \geq 0$. This is impossible because $E$ is stable.

The $H^{0}\left(E \otimes\left(\Omega_{X}^{1}\right)^{k}\right)$ form a holomorphic bundle (see [13]) $A_{k}$ over $S$ of rank $u_{k}$ the dimension of $H^{0}\left(E \otimes\left(\Omega_{X}^{1}\right)^{k}\right)$. By Riemann-Roch, we have

$$
\begin{aligned}
u_{k} & =d\left(E \otimes\left(\Omega_{X}^{1}\right)^{k}\right)+n(1-g) \\
& =d(E)+2 n k(g-1)+n(1-g) \\
& =d+n(g-1)(2 k-1) \\
& =2 h k+d-h \quad(\text { where } h=n(g-1)) .
\end{aligned}
$$

We have

$$
\begin{aligned}
& \left(u_{2}, u_{1}\right)=1 \Leftrightarrow(d+3 h, d+h)=1 \Leftrightarrow(2 h, d+h)=1 \\
& \Leftrightarrow d+h \text { is odd and }(d, h)=1 .
\end{aligned}
$$

As $d$ and $n$ are co prime, $d$ and $h$ are co prime if and only if $d$ and $g-1$ are co prime. If in addition we assume $g-1$ is odd then $d+n(g-1)$ is odd ( $d$ and $n$ have different parities). In this case, there exist integers $a$ and $b$ such that $a u_{1}+b u_{2}=1$ and we can take

$$
L=\left(\wedge^{u_{1}} A_{1}\right)^{a} \otimes\left(\wedge^{u_{2}} A_{2}\right)^{b} .
$$


Otherwise, there exists $g^{\prime} \geq g$ such that $g^{\prime}-1$ is odd and $\left(d, g^{\prime}-1\right)=1$. The injection

$$
\begin{gathered}
\mathbf{S U}(n)^{2 g} \\
\left(A_{1}, B_{1}, \ldots, A_{g}, B_{g}\right) \longmapsto\left(A_{1}, B_{1}, \ldots, A_{g}, B_{g}, 1,1, \ldots, 1,1\right)
\end{gathered}
$$

restricts to an equivariant injection

$$
S \rightarrow S^{\prime}
$$

where $S^{\prime}$ is the set of $2 g^{\prime}$-tuple of matrices

$$
S^{\prime}=\left\{\left(A_{1}, B_{1}, \ldots, A_{g^{\prime}}, B_{g^{\prime}}\right), \prod_{k=1}^{g^{\prime}}\left[A_{k}, B_{k}\right]=\zeta \mathbf{I}\right\} .
$$

We have seen we can construct on $S^{\prime}$ a line bundle with the required properties. We take $L$ to be the restriction of this bundle to $S$.

Let us use the universal bundle to define classes in $H^{*}\left(\mathfrak{m}_{\beta}\right)$.

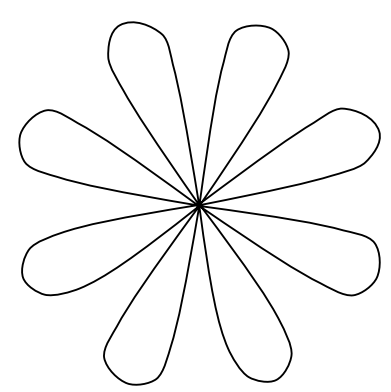

Figure 1. Bouquet of $2 g$ circles (with $g=4$ )

Let $B$ be a bouquet of $2 g$ circles (Figure 1 ) embedded in $X^{\prime}$ in such a way that $X^{\prime}$ retracts on $B$. Each of the $2 g$ circles defines a class in $H_{1}(X)$. Let $\alpha_{1}, \ldots, \alpha_{2 g}$ be their Poincaré duals. They form a basis of $H^{1}(X)$. Let $\kappa$ be the class of the volume form on $X$ of volume 1 . Let us decompose the characteristic classes of the projective bundle $P(U)$. For $k$ in $[2, n]$ :

$$
p_{k}(P(U))=a_{k} \otimes \mathbf{1}+\sum_{j=1}^{2 g} b_{k, j} \otimes \alpha_{j}+d_{k} \otimes \kappa .
$$

Then, according to Biswas and Raghavendra [4], we have 
Theorem 3.4. The family

$$
\left\{a_{k}, b_{k, j}, d_{k}, 2 \leq k \leq n, 1 \leq j \leq 2 g\right\}
$$

is a multiplicative system of generators of $H^{*}\left(\mathfrak{m}_{\beta}\right) \simeq H_{\mathbf{S U}(n)}^{*}\left(\mu^{-1}(\beta)\right)$.

\section{A bundle over $\left(\mathbf{S U}(n)^{2 g}\right)_{\mathrm{SU}(n)} \times X^{\prime}$ and its Chern classes}

Let $B$ be a bouquet of $2 g$ circles (Figure 1 ) embedded in $X^{\prime}$ in such a way that $X^{\prime}$ retracts on $B$. The theory of vector bundles with their Chern classes is the same on $B$ and $X^{\prime}$. We want to construct a complex vector bundle on $\left(\mathbf{S U}(n)^{2 g}\right)_{\mathbf{S U}(n)} \times B$. Denote $B^{\prime}$ the star with $2 g$ branches (see Figure 2 ), that is $B^{\prime}=\left(\cup_{i=1}^{2 g}[0,1]_{i}\right) / \sim$,

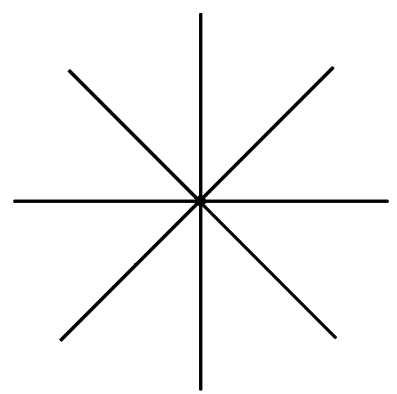

FiguRE 2. A star with $2 g$ branches (with again $g=4$ )

where $\sim$ is the equivalence relation that identifies all the 0 to a point. There is a natural map

$$
\eta: B^{\prime} \longrightarrow B .
$$

It is defined by means of the exponential exp : $[0,1] \rightarrow S^{1}$. Denote

$$
D_{n}=\left(\mathbf{S U}(n)^{2 g} \times E \mathbf{U}(n) \times B^{\prime} \times \mathbf{C}^{n}\right) / \sim
$$

where $\sim$ is the relation:

$$
\begin{gathered}
\left(\left(\rho_{1}, \ldots, \rho_{2 g}\right), e, 0, v\right) \sim\left(\left(\operatorname{Ad}_{A} \rho_{1}, \ldots, \operatorname{Ad}_{A} \rho_{2 g}\right), A \cdot e, 1_{i}, A \circ \rho_{i}(v)\right), \\
\forall i \in[1,2 g], \forall A \in \mathbf{S U}(n) .
\end{gathered}
$$

The projection

$$
D_{n} \longrightarrow\left(\mathbf{S U}(n)^{2 g}\right)_{\mathbf{S U}(n)} \times B
$$

makes $D_{n}$ into a rank $n$ complex vector bundle over $\left(\mathbf{S U}(n)^{2 g}\right)_{\mathbf{S U}(n)} \times B$. We wish to compute the characteristic classes of the projectivised bundle $P\left(D_{n}\right)$ of $D_{n}$. Notice that as the structure group of $D_{n}$ reduces to $\mathbf{S U}(n)$, the classes $p_{k}(P(D))$ are equal to the Chern classes $c_{k}(D)$. 
Let us describe the cohomology of $\left(\mathbf{S U}(n)^{2 g}\right)_{\mathbf{S U}(n)} \times B$. By the Künneth formula, we have

$$
H^{*}\left(\left(\mathbf{S U}(n)^{2 g}\right)_{\mathbf{S U}(n)} \times B\right)=H_{\mathbf{S U}(n)}^{*}\left(\mathbf{S U}(n)^{2 g}\right) \otimes H^{*}(B) .
$$

Proposition 4.1. Let $G$ be a compact Lie group. Let $k$ be an integer bigger than 0 . Let $G$ act on $G^{k}$ diagonally by conjugation. The equivariant cohomology of $G^{k}$ is isomorphic, as a graded algebra, to $H^{*}\left(G^{k}\right) \otimes H^{*}(B G)$.

Proof. The fibration $\left(G^{k}\right)_{G} \longrightarrow B G$ is cohomologically trivial (see [3]) so that we have an isomorphism of graded vector spaces between $H_{G}^{*}\left(G^{k}\right)$ and $H^{*}\left(G^{k}\right) \otimes$ $H^{*}(B G)$. The proposition then follows from the fact that for any compact Lie group, its cohomology is an exterior algebra on a finite number of elements and from the

Lemma 4.2. Let $q: N \longrightarrow M$ be a cohomologically trivial fibration with fiber $F$. Assume that the cohomology of $F$ is an exterior algebra on a family $\left\{\xi_{1}, \ldots, \xi_{r}\right\}$. Thenthe cohomology of $N$ is isomorphic, as a graded algebra, to the tensor product of $H^{*}(F)$ and $H^{*}(M)$.

Proof. Let $\mathfrak{I}$ be the set of strictly increasing sequences of integers $I=\left(i_{1}, \ldots, i_{p}\right)$ such that $i_{1} \geq 1$ and $i_{p} \leq r$. For $I \in \mathfrak{I}$ with $I=\left(i_{1}, \ldots, i_{p}\right)$, let

$$
\xi_{I}=\xi_{i_{1}} \wedge \cdots \wedge \xi_{i_{p}}
$$

The family $\left\{\xi_{I}\right\}_{I \in \mathfrak{I}}$ forms a basis of $H^{*}(F)$.

To say that the fibration $N \longrightarrow M$ is cohomologically trivial is equivalent (by the Leray-Hirsch Theorem) to saying that the inclusion of a fiber $F$ into $N$ induces a surjection $H^{*}(N) \longrightarrow H^{*}(F)$. For $i \in[1, r]$, let $\zeta_{i}$, in $H^{*}(N)$, be a pre-image of $\xi_{i}$. For $I \in \mathfrak{I}$ with $I=\left(i_{1}, \ldots, i_{p}\right)$, let

$$
\zeta_{I}=\zeta_{i_{1}} \wedge \cdots \wedge \zeta_{i_{p}}
$$

The map

$$
\begin{aligned}
& H^{*}(F) \longrightarrow H^{*}(N) \\
& \sum \lambda_{I} \zeta_{I} \longmapsto \sum \lambda_{I} \xi_{I}
\end{aligned}
$$

is a morphism of algebra and the map

$$
\begin{gathered}
H^{*}(F) \otimes H^{*}(M) \\
\left(\sum \lambda_{I} \zeta_{I}\right) \otimes \chi \\
\quad \longmapsto\left(\sum \lambda_{I} \xi_{I}\right) \otimes q^{*}(\chi)
\end{gathered}
$$

is an isomorphism of graded algebra. 
According to the previous proposition, we have isomorphisms

$$
\begin{aligned}
H_{\mathbf{S U}(n)}^{*}\left(\mathbf{S U}(n)^{2 g}\right) & \simeq H^{*}\left(\mathbf{S U}(n)^{2 g}\right) \otimes H^{*}(B \mathbf{S U}(n)) \\
& \simeq \otimes_{j=1}^{2 g} H^{*}(\mathbf{S U}(n)) \otimes H^{*}(B \mathbf{S U}(n)) .
\end{aligned}
$$

For all $k \geq 2$, the fibration $\mathbf{S U}(k) \longrightarrow S^{2 k-1}$ with fiber $\mathbf{S U}(k-1)$ is cohomologically trivial (see Hatcher [7]). Let $\gamma_{k}$ be the volume form of volume 1 on $S^{2 k-1}$. The cohomology of $\mathbf{S U}(n)$ is the exterior algebra freely generated by the family $\left\{\sigma_{k}, 2 \leq k \leq n\right\}$, where $\sigma_{k}$ is a class of degree $2 k-1$ which pulls-back under the restriction $\mathbf{S U}(k) \longrightarrow \mathbf{S U}(n)$ to the image of $\gamma_{k}$ by $H^{2 k-1}\left(S^{2 k-1}\right) \longrightarrow$ $H^{2 k-1}(\mathbf{S U}(k))$. Denote $\sigma_{k, j}$ the image of $\sigma_{k} \in H^{2 k-1}(\mathbf{S U}(n))$ by the homomorphism $H^{*}(\mathbf{S U}(n)) \rightarrow H^{*}\left(\mathbf{S U}(n)^{2 g}\right)$ induced by the projection on the $j$-th factor $\mathbf{S U}(n)^{2 g} \rightarrow \mathbf{S U}(n)$. We have

Lemma 4.3. The algebra $H^{*}\left(\mathbf{S U}(n)^{2 g}\right)$ is the exterior algebra freely generated by the family $\left\{\sigma_{k, j}, 2 \leq k \leq n, 1 \leq j \leq 2 g\right.$, deg $\left.\sigma_{k, j}=2 k-1\right\}$.

In addition, we know that $H^{*}(B \mathbf{S U}(n))=\mathbf{Q}\left[c_{2}, \ldots, c_{n}\right]$. From the preceding lemma and Proposition 4.1, we deduce

Theorem 4.4. Let $\Lambda$ be the exterior algebra freely generated by the family $\left\{\sigma_{k, j}\right.$, $2 \leq k \leq n, 1 \leq j \leq 2 g$, deg $\left.\sigma_{k, j}=2 k-1\right\}$. The $\mathbf{S U}(n)$-equivariant cohomology of $\mathbf{S U}(n)^{2 g}$ is isomorphic, as a graded algebra, to $\Lambda \otimes \mathbf{Q}\left[c_{2}, \ldots, c_{n}\right]$.

When there is no risk of confusion, we will write $c_{k}$ and $\sigma_{k, j}$ instead of respectively $1 \otimes c_{k}$ and $\sigma_{k, j} \otimes 1$.

Remark 4.5. The injection $\iota$ of $\mathbf{S U}(n)$ into $\mathbf{S U}(n+1)$ and the map $B \mathbf{S U}(n) \rightarrow$ $B \mathbf{S U}(n+1)$ induce isomorphisms

$$
H^{k}(\mathbf{S U}(n+1)) \stackrel{\sim}{\longrightarrow} H^{k}(\mathbf{S U}(n)) \text { for } k \leq 2 n \text { and } k=2 n+2
$$

and

$$
H^{k}(B \mathbf{S U}(n+1)) \stackrel{\sim}{\longrightarrow} H^{k}(B \mathbf{S U}(n)) \text { for } k \leq 2 n .
$$

With the notations of Theorem 4.4, we have

Proposition 4.6. The Chern classes of $D_{n}$ are:

$$
\begin{aligned}
& c_{0}\left(D_{n}\right)=1, \\
& c_{1}\left(D_{n}\right)=0, \\
& c_{k}\left(D_{n}\right)=\left(1 \otimes c_{k}\right) \otimes 1+\sum_{j=1}^{2 g}\left(\sigma_{k, j} \otimes 1\right) \otimes \alpha_{j} \text { for } k \geq 2 .
\end{aligned}
$$

Proof. The classes $c_{0}\left(D_{n}\right)$ and $c_{1}\left(D_{n}\right)$ are trivially 1 and 0 (the structure group is $\mathbf{S U}(n))$. Assume from now on that $k \geq 2$. Let us write the Chern classes of $D_{n}$ 
in $H^{*}\left(\left(\mathbf{S U}(n)^{2 g}\right)_{\mathbf{S U}(n)} \times B\right)$ as

$$
c_{k}\left(D_{n}\right)=\gamma_{k}^{(n)} \otimes 1+\sum_{j=1}^{2 g} \beta_{k, j}^{(n)} \otimes \alpha_{j}
$$

We will prove the proposition by induction on $n$. For $n=1, \mathbf{S U}(1)$ is just a point, the bundle $D_{1}$ is trivial and we are already done. Suppose the proposition to be true for a given $n, n \geq 1$ and let us prove it for $n+1$. We need to prove that

$$
\gamma_{k}^{(n+1)}=1 \otimes c_{k} \text { and } \beta_{k, j}^{(n+1)}=\sigma_{k, j} \otimes 1
$$

Let

$$
m:\left(\mathbf{S U}(n)^{2 g}\right)_{\mathbf{S U}(n)} \longrightarrow\left(\mathbf{S U}(n+1)^{2 g}\right)_{\mathbf{S U}(n+1)},
$$

the map induced by the inclusion $\mathbf{S U}(n) \longrightarrow \mathbf{S U}(n+1)$ and

$$
\ell=m \times \operatorname{id}_{B}:\left(\mathbf{S U}(n)^{2 g}\right)_{\mathbf{S U}(n)} \times B \longrightarrow\left(\mathbf{S U}(n+1)^{2 g}\right)_{\mathbf{S U}(n+1)} \times B .
$$

The bundle $\ell^{*} D_{n+1}$ is isomorphic to $D_{n} \oplus \mathbf{C}$. Hence, for all $k$, we have $c_{k}\left(\ell^{*} D_{n+1}\right)=$ $c_{k}\left(D_{n}\right)$. Thus

$$
\left(m^{*} \gamma_{k}^{(n+1)}\right) \otimes 1+\sum_{j=1}^{2 g}\left(m^{*} \beta_{k, j}^{(n+1)}\right) \otimes \alpha_{j}=\gamma_{k}^{(n)} \otimes 1+\sum_{j=1}^{2 g} \beta_{k, j}^{(n)} \otimes \alpha_{j} .
$$

From this we deduce

$$
m^{*} \gamma_{k}^{(n+1)}=\gamma_{k}^{(n)} \text { and } m^{*} \beta_{k, j}^{(n+1)}=\beta_{k, j}^{(n)}
$$

Because of the isomorphisms (4.1), (4.2) and the induction hypothesis, we have:

$$
\begin{aligned}
& \gamma_{k}^{(n+1)}=1 \otimes c_{k} \text { for } k \leq n, \\
& \beta_{k, j}^{(n+1)}=\sigma_{k, j} \otimes 1 \text { for } k \leq n .
\end{aligned}
$$

There only remains to compute $\gamma_{n+1}^{(n+1)}$ and the $\beta_{n+1, j}^{(n+1)}$. The class $\gamma_{n+1}^{(n+1)}$ belongs to

$$
H_{\mathbf{S U}(n+1)}^{2 n+2}\left(\mathbf{S U}(n+1)^{2 g}\right)=\bigoplus_{p+q=2 n+2} H^{p}\left(\mathbf{S U}(n+1)^{2 g}\right) \otimes H^{q}(B \mathbf{S U}(n+1)) .
$$

Let us decompose it

$$
\gamma_{n+1}^{(n+1)}=\sum_{k=0}^{n+1} \varepsilon_{k}^{(n+1)} \otimes c_{k}
$$

where $\varepsilon_{k}^{(n+1)}$ is in $H^{2 n+2-2 k}\left(\mathbf{S U}(n+1)^{2 g}\right)$ and where we have put $c_{0}=1$ in $H^{0}(B \mathbf{S U}(n)), c_{1}=0$. The classes $\beta_{n+1, j}^{(n+1)}$ are in

$$
H_{\mathbf{S U}(n+1)}^{2 n+1}\left(\mathbf{S U}(n+1)^{2 g}\right)=\bigoplus_{p+q=2 n+1} H^{p}\left(\mathbf{S U}(n+1)^{2 g}\right) \otimes H^{q}(B \mathbf{S U}(n+1))
$$


We decompose them in

$$
\beta_{n+1, j}^{(n+1)}=\sum_{k=0}^{n} \delta_{k, j}^{(n+1)} \otimes c_{k}
$$

where $\delta_{k, j}^{(n+1)}$ belongs to $H^{2 n+1-2 k}\left(\mathbf{S U}(n+1)^{2 g}\right)$. The bundle $\ell^{*} D_{n+1}=D_{n} \oplus \mathbf{C}$ has a nowhere vanishing section, hence its Euler class $\ell^{*} c_{n+1}\left(D_{n+1}\right)$ vanishes. Because of the isomorphisms (4.1) and (4.2), we deduce that the $\left\{\varepsilon_{k}^{(n+1)}, 1 \leq\right.$ $k \leq n\}$ and the $\left\{\delta_{k, j}^{(n+1)}, 1 \leq k \leq n, 1 \leq j \leq 2 g\right\}$ vanish. Remark that the $\left\{\delta_{n+1, j}^{(n+1)}, 1 \leq j \leq 2 g\right\}$ are linear combinations of the $\sigma_{2 n+1, j}, 1 \leq j \leq 2 g$. Let us define a section

$$
\begin{aligned}
& s: B \mathbf{S U}(n+1) \longrightarrow\left(\mathbf{S U}(n+1)^{2 g}\right)_{\mathbf{S U}(n+1)} \times B \\
& {[e] \quad \longmapsto \quad([(\mathbf{I}, \ldots, \mathbf{I}), e], 1)}
\end{aligned}
$$

where, for $e$ in $E \mathbf{U}(n+1)$, we denote by $[e]$ its class in $B \mathbf{S U}(n+1)$. The Euler class of the bundle $s^{*} D_{n+1}$ is $\varepsilon X_{n+1}$. Since $s^{*} D_{n+1}$ is equal to $E \mathbf{U}(n+1) \times_{\mathbf{S U}(n+1)}$ $\mathbf{C}^{n+1}$ we have $\varepsilon=1$. As a conclusion we have

$$
\gamma_{n+1}^{(n+1)}=1 \otimes X_{n+1} .
$$

Let

$$
h: \mathbf{S U}(n+1)^{2 g} \longrightarrow\left(\mathbf{S U}(n+1)^{2 g}\right)_{\mathbf{S U}(n+1)}
$$

be the inclusion of a fiber (we will always write $h$ this application, omitting the subscript $n$ ). The bundle

$$
F_{n+1}^{2 g}:=\left(h \times \mathrm{id}_{B}\right)^{*} D_{n+1}
$$

is isomorphic to

$$
F_{n+1}^{2 g} \cong\left(\mathbf{S U}(n+1)^{2 g} \times B^{\prime} \times \mathbf{C}^{n+1}\right) / \sim,
$$

where $\sim$ is the relation:

$$
\left(\left(\rho_{1}, \ldots, \rho_{2 g}\right), 1_{j}, v\right) \sim\left(\left(\rho_{1}, \ldots, \rho_{2 g}\right), 0, \rho_{j}^{-1}(v)\right), \text { for all } j \text { in }[1,2 g] .
$$

The Euler class of $F_{n+1}^{2 g}$ is

$$
c_{n+1}\left(F_{n+1}^{2 g}\right)=\sum_{j=1}^{2 g} \beta_{n+1, j}^{(n+1)} \otimes \alpha_{j} .
$$

Let $f_{j}: S^{1} \rightarrow B$ (resp. $\left.g_{j}: \mathbf{S U}(n+1) \rightarrow \mathbf{S U}(n+1)^{2 g}\right)$ be the inclusion of the $j$-th circle (resp. $\mathbf{S U}(n+1))$ in $B$ (resp. $\left.\mathbf{S U}(n+1)^{2 g}\right)$. The $\beta_{n+1, j}^{(n+1)}$ are characterised by:

$$
c_{n+1}\left(\left(\operatorname{id}_{\mathbf{S U}(n+1)^{2 g}} \times f_{j}\right)^{*} F_{2 g}^{(n+1)}\right)=\beta_{n+1, j}^{(n+1)} \otimes f_{j}^{*} \alpha_{j},
$$

or

$$
c_{n+1}\left(\left(\operatorname{id}_{\mathbf{S U}(n+1)^{2 g}} \times f_{j}\right)^{*} F_{2 g}^{(n+1)}\right)=\beta_{n+1, j}^{(n+1)} \otimes \frac{\mathrm{d} \theta}{2 \pi} .
$$


Let us define a vector bundle $E$ over $\mathbf{S U}(n+1) \times S^{1}$ by

$$
E=(\mathbf{S U}(n+1) \times[0,1] \times \mathbf{C}) / \sim,
$$

where $\sim$ is the relation

$$
(\rho, 1, v) \sim\left(\rho, 0, \rho^{-1}(v)\right) .
$$

The bundle $\left(\operatorname{id}_{\mathbf{S U}(n+1)^{2 g}} \times f_{j}\right)^{*} F_{2 g}^{(n+1)}$ is isomorphic to $\left(g_{j} \times \mathrm{id}_{S^{1}}\right)^{*} E$. Hence there exists a real $\lambda$ such that

$$
c_{n+1}(E)=\lambda \sigma_{2 n+1} \otimes \frac{\mathrm{d} \theta}{2 \pi} .
$$

If $(\rho, t, v)$ belongs to $\mathbf{S U}(n+1) \times[0,1] \times \mathbf{C}^{n+1}$, let us write $[\rho, t, v]$ for its class in $E$. Let $\left(e_{1}, \ldots, e_{n+1}\right)$ be the canonical basis, over the field $\mathbf{C}$, of $\mathbf{C}^{n+1}$. The family $\left(e_{1}, i e_{1}, \ldots, e_{n+1}, i e_{n+1}\right)$ is then a basis of $\mathbf{C}^{n+1}$ over $\mathbf{R}$. A section of $E$ is given by:

$$
\begin{aligned}
& s: \mathbf{S U}(n+1) \times S^{1} \longrightarrow \quad E \\
& \left(A, e^{2 i \pi \theta}\right) \longmapsto\left[A, \theta,(\theta A+(1-\theta) \mathrm{id}) e_{1}\right] \text {. }
\end{aligned}
$$

Let us determine its zeros. The vector $\left(\theta A+(1-\theta)\right.$ id) $e_{1}$ vanishes if $\theta=\frac{1}{2}$ and $A=\left[\begin{array}{cc}-1 & 0 \\ 0 & \widetilde{A}\end{array}\right], \widetilde{A} \in \mathbf{U}(n), \operatorname{det} \widetilde{A}=-1$. Fix $\xi$ an $n$-th root of -1 . The zero set $Z$ of $s$ is

$$
Z=\left\{\left(\left[\begin{array}{cc}
-1 & 0 \\
0 & \xi \widetilde{A}
\end{array}\right], \frac{1}{2}\right), \widetilde{A} \in \mathbf{S U}(n)\right\}
$$

Lemma 4.7. The section $s$ intersects the zero section $s_{0}$ transversally.

Proof. We want to prove that for all $x$ of $Z$

$$
T_{s(x)} \operatorname{Im} s+T_{s(x)} \operatorname{Im} s_{0}=T_{(x, 0)} E .
$$

We have

$$
T_{(x, 0)} E \simeq T_{x}\left(\mathbf{S U}(n+1) \times S^{1}\right) \oplus \mathbf{C}^{n+1} \simeq \mathfrak{s u}(n+1) \oplus \mathbf{R} \oplus \mathbf{C}^{n+1}
$$

and

$$
\begin{aligned}
& T_{s(x)} \operatorname{Im} s_{0}=\mathfrak{s u}(n+1) \oplus \mathbf{R} \oplus\{0\}, \\
& T_{s(x)} \operatorname{Im} s=\mathrm{T}_{x} s\left(T_{x}\left(\mathbf{S U}(n+1) \times S^{1}\right)\right) .
\end{aligned}
$$

Let $x$ be the point $\left(A=\left[\begin{array}{cc}-1 & 0 \\ 0 & \xi \widetilde{A}\end{array}\right], \frac{1}{2}\right)$,

$$
\begin{aligned}
\left.\frac{d}{d \varepsilon}\right|_{\varepsilon=0} s\left(A, \frac{1}{2}+\varepsilon\right) & =\left[A, \frac{1}{2}+\varepsilon,\left(\left(\frac{1}{2}+\varepsilon\right) A+\left(\frac{1}{2}-\varepsilon\right) \mathrm{id}\right) e_{1}\right] \\
& =\left[A, \frac{1}{2}+\varepsilon,-2 \varepsilon e_{1}\right] \\
& =\left(0,1,-2 e_{1}\right) .
\end{aligned}
$$


Let $J$ be in $\mathfrak{s u}(n+1)$,

$$
\begin{aligned}
\left.\frac{d}{d \varepsilon}\right|_{\varepsilon=0} s\left(\exp (\varepsilon J) A, \frac{1}{2}\right) & =\left.\frac{d}{d \varepsilon}\right|_{\varepsilon=0}\left[\exp (\varepsilon J) A, \frac{1}{2}, \frac{1}{2}(\exp (\varepsilon J) A+\mathrm{id}) e_{1}\right] \\
& =\left.\frac{d}{d \varepsilon}\right|_{\varepsilon=0}\left[\exp (\varepsilon J) A, \frac{1}{2}, \frac{1}{2}\left(\exp (\varepsilon J)\left(-e_{1}\right)+e_{1}\right)\right] \\
& =\left(J \cdot A, 0, \frac{1}{2}\left(-J e_{1}+e_{1}\right)\right) .
\end{aligned}
$$

We conclude the proof of Lemma 4.7 by noticing that, for any $k$, it is possible to find $J$ in $\mathfrak{s u}(n+1)$ such that $J e_{1}$ is equal to $e_{k}$ or $i e_{k}$.

Lemma 4.8. The Euler class of the bundle $E$ is

$$
c_{n+1}(E)=\sigma_{2 n+1} \otimes \frac{\mathrm{d} \theta}{2 \pi} .
$$

Proof. According to the preceding lemma, the Euler class of $E$ is Poincaré dual of $Z$, that is it is characterised by

$$
\forall \nu \in H^{n^{2}-1}\left(\mathbf{S U}(n+1) \times S^{1}\right), \int_{Z} \nu=\int_{\mathbf{S U}(n+1) \times S^{1}} \nu \wedge c_{n+1}(E)
$$

where $n^{2}-1=\operatorname{dim}\left(\mathbf{S U}(n+1) \times S^{1}\right)-2(n+1)$. This Euler class is of the type

$$
c_{n+1}(E)=\lambda \sigma_{2 n+1} \otimes \frac{\mathrm{d} \theta}{2 \pi}
$$

where $\lambda$ is a real we are going to compute. The injection

$$
\begin{aligned}
\mathbf{S U}(n) & \longrightarrow \mathbf{S U}(n+1) \\
A & \longmapsto\left[\begin{array}{cc}
-1 & 0 \\
0 & \xi A
\end{array}\right]
\end{aligned}
$$

identifies $\mathbf{S U}(n)$ to the fibre above $(-1,0, \ldots, 0)$ of the projection $\mathbf{S U}(n+1) \rightarrow$ $S^{2 n+1}$, that is $Z$. Let $\gamma$ be the cohomology class of a volume form of volume 1 over $\mathbf{S U}(n)$. The decomposition $H^{*}(\mathbf{S U}(n+1))=H^{*}(\mathbf{S U}(n)) \otimes H^{*}\left(S^{2 n+1}\right)$ defines a class

$$
\nu=\gamma \otimes 1
$$

As the integral of $\nu$ on $Z$ is 1 , we have

$$
\int_{\mathbf{S U}(n+1) \times S^{1}} \nu \wedge c_{n+1}(E)=1
$$

that is

$$
\lambda \int_{\mathbf{S U}(n+1) \times S^{1}}(\gamma \otimes 1) \wedge\left(\sigma_{2 n+1} \otimes \frac{\mathrm{d} \theta}{2 \pi}\right)=1 .
$$

The conclusion follows since the integral in the left-hand side of the equality is equal to 1 .

Proposition 4.6 follows from this lemma. 


\section{Description of the restriction map}

Using results of the previous sections, we wish to prove:

Theorem 5.1. The restriction map $r$ is described by

$$
\begin{aligned}
r\left(c_{k}\right) & =a_{k} \text { for } k=2, \ldots, n \\
r\left(\sigma_{k, j}\right) & =b_{k, j} \text { for } k=2, \ldots, n, j=1, \ldots, 2 g .
\end{aligned}
$$

In particular, $\operatorname{Im}(r)$ is multiplicatively generated by

$$
\operatorname{Im}(r)=\left\langle a_{k}, b_{k, j}, k=2, \ldots, n, j=1, \ldots, 2 g\right\rangle .
$$

Notice that for $n$ equals 2, we get that $r$ is surjective modulo the symplectic form on $\mathfrak{m}_{\beta}$ (this result has been in [18]).

It is also very interesting to compare this theorem with [11, Theo. 7.1] where a group cohomological construction of multiplicative generators of $H^{*}\left(\mathfrak{m}_{\beta}\right)$ is given.

Proof. The key point of the proof is to compare the bundles $U$ of Section 3 and $D_{n}$ of Section 4.

From now on, if $g \in \mathbf{S U}(n)$, we denote $\bar{g}$ its class in $\mathbf{P S U}(n)$. Over each $S \times U_{i}$, $i=0, \ldots, m$, the bundle $M \otimes T \otimes L^{*}$ is trivial. In each of these sets, the action of $\operatorname{PSU}(n)$ on $M \otimes T \otimes L^{*}$ is

$$
\begin{aligned}
\operatorname{PSU}(n) \times M \otimes\left(S \times U_{i} \times \mathbf{C}^{n}\right) \otimes L^{*} & \longrightarrow M \otimes\left(S \times U_{i} \times \mathbf{C}^{n}\right) \otimes L^{*} \\
m \otimes(\bar{g},(\rho, x, u) \otimes l) & \longmapsto m \otimes(g \cdot \rho, x, g(u)) \otimes(g \cdot l) .
\end{aligned}
$$

Lemma 5.2. We have

$$
\mathrm{P}(U)=\mathrm{P}\left(M \otimes\left(T \otimes L^{*}\right) / \mathbf{P S U}(n)\right) \cong \mathrm{P}(T) / \mathbf{P S U}(n) .
$$

Proof. This time, $\mathbf{P S U}(n)$ acts on $\mathrm{P}(T)$ by

$$
\begin{aligned}
& \operatorname{PSU}(n) \times\left(S \times U_{i} \times \mathbf{C P}^{n}\right) \longrightarrow\left(S \times U_{i} \times \mathbf{C P}^{n}\right) \\
& (\bar{g},(\rho, x, \bar{u})) \quad \longmapsto(g \cdot \rho, x, \overline{g(u)})
\end{aligned}
$$

and the announced isomorphism is

$$
\begin{aligned}
& \mathrm{P}(U) \cong \\
& \text { class of } m \otimes(\rho, x, u) \otimes l \longmapsto \\
& \mathrm{P}(T) / \mathbf{P S U}(n) \\
& \text { class of }(\rho, x, u) .
\end{aligned}
$$

Lemma 5.3. There exists an action of $\pi \times \mathbf{P S U}(n)$ on $S \times Y^{\prime} \times \mathbf{C P}^{n-1}$ such that the quotient

$$
\left(S \times Y^{\prime} \times \mathbf{C P}^{n-1}\right) /(\pi \times \mathbf{P S U}(n))
$$

is isomorphic to

$$
\left.\mathrm{P}(U)\right|_{\mathfrak{m}_{\beta} \times X^{\prime}} .
$$


Proof. The bundle $T$ restricted to $S \times X^{\prime}$ is trivial on each $S \times U_{i}, i \neq 0$ and transition functions are given by

$$
\begin{aligned}
\left(S \times U_{i}\right) \cap\left(S \times U_{j}\right) & \longrightarrow \mathbf{S U}(n) \\
(\rho, x) & \longmapsto \rho\left(\gamma_{i, j}\right) .
\end{aligned}
$$

The group $\pi$ acts freely on $Y^{\prime}$ and $\left.T\right|_{S \times X^{\prime}}$ is $\left(S \times Y^{\prime} \times \mathbf{C}^{n}\right) / \pi$, where the action of $\pi$ is

$$
\begin{aligned}
\pi \times\left(S \times Y^{\prime} \times \mathbf{C}^{n}\right) & \longrightarrow S \times Y^{\prime} \times \mathbf{C}^{n} \\
(\gamma,(\rho, y, u)) & \longmapsto(\rho, \gamma \cdot y, \rho(\gamma) u) .
\end{aligned}
$$

Let us consider the projective bundle $\left.\mathrm{P}(T)\right|_{S \times X^{\prime}}$. It is isomorphic to $\left(S \times Y^{\prime} \times\right.$ $\left.\mathbf{C P}^{n-1}\right) / \pi$. The subspace $\left.\mathrm{P}(T)\right|_{S \times X^{\prime}}$ is stable by $\mathbf{P S U}(n)$ and the action comes from an action of $\mathbf{P S U}(n)$ on $S \times Y^{\prime} \times \mathbf{C P}^{n-1}$. That is

$$
\begin{aligned}
& \mathbf{P S U}(n) \times\left(S \times Y^{\prime} \times \mathbf{C P}^{n-1}\right) \longrightarrow S \times Y^{\prime} \times \mathbf{C P}^{n-1} \\
& (\bar{g},(\rho, y, \bar{u})) \quad \longmapsto(g \cdot \rho, y, \overline{g(u)}) .
\end{aligned}
$$

This action commutes indeed with the one of $\pi$, the result follows.

The pull-back of the bundle $U \rightarrow(S / \mathbf{P S U}(n)) \times X^{\prime}$ to $(S)_{\mathbf{S U}(n)} \times X^{\prime}$ by the natural map

$$
f:(S)_{\mathbf{S U}(n)} \times X^{\prime} \longrightarrow(S / \mathbf{P S U}(n)) \times X^{\prime}
$$

is a vector bundle, we will denote it $F$. Its projectivised bundle is

$$
\mathrm{P}(F)=(\mathrm{P}(T))_{\mathbf{S U}(n)} \longrightarrow(S)_{\mathbf{S U}(n)} \times X^{\prime} .
$$

We will now state a proposition which will be our main tool in the study of the map $r$ :

Proposition 5.4. There is a projective bundle $\mathrm{P}(D)$ over $\left(\mathbf{S U}(n)^{2 g}\right)_{\mathbf{S U}(n)} \times X^{\prime}$ whose restriction to $(S)_{\mathbf{S U}(n)} \times X^{\prime}$ is isomorphic to $\mathrm{P}(F)$.

First proof. The projection $p: Y^{\prime} \rightarrow X^{\prime}$ is a covering. Its group is $\pi$. Let $q: \widetilde{Y}^{\prime} \rightarrow Y^{\prime}$ be the universal covering of $Y^{\prime}$. The composed map $\widetilde{p}=p \circ q: \widetilde{Y}^{\prime} \rightarrow X^{\prime}$ is the universal covering of $X^{\prime}$. Its group is

$$
\pi_{1}\left(X^{\prime}\right)=\left\langle a_{1}, b_{1}, \ldots, a_{g}, b_{g}\right\rangle
$$

and we have a projection $\pi_{1}\left(X^{\prime}\right) \stackrel{\theta}{\rightarrow} \pi$ whose kernel is the group of the covering $\tilde{Y}^{\prime} \rightarrow Y^{\prime}$.

The open covering of $X^{\prime}$ by the $\left\{U_{i}\right\}_{i=1}^{m}$ is such that any intersection of open sets of the type $U_{i}$ is contractible. In particular, for all $i$, there exists a disc $\widetilde{D}_{i}$ in $\widetilde{Y}^{\prime}$ such that $\widetilde{p}: \widetilde{D}_{i} \rightarrow U_{i}$ is a diffeomorphism. Choose, for all $i, j, k$, a connected component $W_{i j, k}$ of $\widetilde{p}^{-1}\left(U_{i} \cap U_{j}\right) \cap \widetilde{D}_{k}$. If $U_{i} \cap U_{j} \neq \emptyset$, let $\widetilde{\gamma}_{i, j}$ be the element of $\pi_{1}\left(X^{\prime}\right)$ such that $\widetilde{\gamma}_{i, j} \widetilde{W}_{i j, j}=\widetilde{W}_{j i, i}$. In Proposition 2.2, we can take the $W_{i j, k}$ and $\gamma_{i, j}$ such that

$$
W_{i j, k}=\widetilde{p}\left(\widetilde{W}_{i j, k}\right), \gamma_{i, j}=\theta\left(\widetilde{\gamma}_{i, j}\right) .
$$


Let us identify the set of representations $\rho: \pi_{1}\left(X^{\prime}\right) \rightarrow \mathbf{S U}(n)$ to $\mathbf{S U}(n)^{2 g}$ by

$$
\rho \mapsto\left(\rho\left(a_{1}\right), \rho\left(b_{1}\right), \ldots, \rho\left(a_{g}\right), \rho\left(b_{g}\right)\right) .
$$

Let

$$
T^{\prime} \rightarrow \mathbf{S U}(n)^{2 g} \times X^{\prime}
$$

be the rank $n$ complex vector bundle defined by the following properties:

(1) $\left.T^{\prime}\right|_{\mathbf{S U}(n)^{2 g} \times U_{i}}$ is trivial,

(2) the transition functions are

$$
g_{i, j}=\rho\left(\widetilde{\gamma}_{i, j}\right) \text { on } \mathbf{S U}(n)^{2 g} \times\left(U_{i} \cap U_{j}\right) .
$$

The restriction of this bundle to $S \times X^{\prime}$ is $\left.T\right|_{S \times X^{\prime}}$. The action of $\mathbf{S U}(n)$ on $\left.T\right|_{S \times X^{\prime}}$ is then the restriction of the $\mathbf{S U}(n)$ action on $T^{\prime}$ defined on each $\left.T^{\prime}\right|_{\mathbf{S U}(n)^{2 g} \times U_{i}}$ by

$$
\begin{aligned}
\mathbf{S U}(n) \times\left(\mathbf{S U}(n)^{2 g} \times U_{i} \times \mathbf{C}^{n}\right) & \longrightarrow \mathbf{S U}(n)^{2 g} \times U_{i} \times \mathbf{C}^{n} \\
(g,(\rho, x, u)) & \longmapsto(g \cdot \rho, x, g(u)) .
\end{aligned}
$$

Notice that this action is a lift of the action of $\mathbf{S U}(n)$ on $\mathbf{S U}(n)^{2 g} \times X^{\prime}$. Thus the bundle

$$
\mathrm{P}(F)=(\mathrm{P}(T))_{\mathbf{S U}(n)} \rightarrow(S)_{\mathbf{S U}(n)} \times X^{\prime}
$$

is the restriction of the bundle

$$
\left(\mathrm{P}\left(T^{\prime}\right)\right)_{\mathbf{S U}(n)} \rightarrow\left(\mathbf{S U}(n)^{2 g}\right)_{\mathbf{S U}(n)} \times X^{\prime} .
$$

Second proof. We have seen that

$$
\left.\mathrm{P}(U)\right|_{\mathfrak{m}_{\beta} \times X^{\prime}} \cong\left(S \times Y^{\prime} \times \mathbf{C P}^{n-1}\right) /(\pi \times \mathbf{P S U}(n)),
$$

hence

$$
\mathrm{P}(F) \cong\left(S \times E \mathbf{U}(n) \times Y^{\prime} \times \mathbf{C P}^{n-1}\right) /(\pi \times \mathbf{S U}(n))
$$

Let us define, in a similar way as before, an action of $\pi_{1}\left(X^{\prime}\right)$ on $\mathbf{S U}(n)^{2 g} \times E \mathbf{U}(n) \times$ $\widetilde{Y^{\prime}} \times \mathbf{C}^{n}$ and denote $D$ the bundle we obtain when quotienting by $\pi_{1}\left(X^{\prime}\right) \times \mathbf{S U}(n)$. The projection $S \times E \mathbf{U}(n) \times \widetilde{Y^{\prime}} \times \mathbf{C}^{n} \rightarrow S \times E \mathbf{U}(n) \times Y^{\prime} \times \mathbf{C}^{n}$ is equivariant for the respective actions of $\pi_{1}\left(X^{\prime}\right)$ and $\pi$. It induces an action on the quotient and defines an isomorphism between

$$
\left(S \times E \mathbf{U}(n) \times \widetilde{Y^{\prime}} \times \mathbf{C}^{n}\right) /\left(\pi_{1}\left(X^{\prime}\right) \times \mathbf{S U}(n)\right)
$$

and

$$
\left(S \times E \mathbf{U}(n) \times Y^{\prime} \times \mathbf{C}^{n}\right) /(\pi \times \mathbf{S U}(n)) .
$$

We deduce that $\mathrm{P}(F)$ is isomorphic to $\left.\mathrm{P}(D)\right|_{(S)_{\mathrm{SU}(n)} \times X^{\prime}}$.

Remark 5.5. The bundle $D \rightarrow\left(\mathbf{S U}(n)^{2 g}\right)_{\mathbf{S U}(n)} \times X^{\prime}$ is isomorphic to $\left(T^{\prime} \times\right.$ $E \mathbf{U}(n)) / \mathbf{S U}(n)$. 
When restricted to $\left(\mathbf{S U}(n)^{2 g}\right)_{\mathbf{S U}(n)} \times B$, the bundle $D$ is isomorphic to $D_{n}$ (restricted to $\left.\left(\mu^{-1}(\zeta \mathbf{I})\right)_{\mathbf{S U}(n)} \times B\right)$. Denote $w$ the injection of $(S)_{\mathbf{S U}(n)} \times X^{\prime}$ in $\left(\mathbf{S U}(n)^{2 g}\right)_{\mathbf{S U}(n)} \times X^{\prime}$. The induced map $w^{*}$ in cohomology is $r \times \operatorname{id}_{H^{*}\left(X^{\prime}\right)}$. The restriction $w^{*} D_{n}$ of $D_{n}$ to $(S)_{\mathbf{S U}(n)} \times X^{\prime}$ has the same projectivisation as $F$. Thus, because of Proposition 4.6, we have for every $k$

$$
\begin{aligned}
p_{k}(P(F)) & =a_{k} \otimes 1+\sum_{j=1}^{2 g} b_{k}^{j} \otimes \alpha_{j} \\
& =p_{k}\left(P\left(w^{*} D_{n}\right)\right) \\
& =w^{*} p_{k}\left(P\left(D_{n}\right)\right) \\
& =r\left(1 \otimes p_{k}\right) \otimes 1+\sum_{j=1}^{2 g} r\left(\sigma_{k, j} \otimes 1\right) \otimes \alpha_{j} .
\end{aligned}
$$

Theorem 5.1 follows from the comparison of Line (5.1) and Line (5.2).

\section{References}

[1] M. F. Atiyah and R. Bott, The Yang-Mills Equations Over Riemann Surfaces, Phil. Trans. Royal Soc. Lond. A308 (1982), 523-615.

[2] A. Alekseev, A. Malkin and E. Meinrenken, Lie group valued moment maps, J. Differential Geom. 48 (1998), 445-495.

[3] A. Alekseev, E. Meinrenken and C. Woodward, Group-Valued Equivariant Localization, Invent. Math. 140 no. 2 (2000), 327-350.

[4] I. Biswas and N. Raghavendra, Canonical generators of the cohomology of moduli of parabolic bundles on curve, Math. Ann. 306 (1996), 1-14.

[5] A. Grothendieck Sur la mémoire de Weil "Généralisation des fonctions abéliennes", Séminaire Bourbaki, Exposé 141, 1956-57.

[6] V. W. Guillemin and S. Sternberg, Supersymmetry and Equivariant de Rham Theory, Mathematics Past and Present, Springer-Verlag, 1999.

[7] A. Hatcher, Algebraic Topology, Cambridge University Press, 2002.

[8] J. Huebschmann, Symplectic and Poisson structures of certain moduli spaces, Duke Math. J. 80 (1995), 737-756.

[9] J. Huebschmann and L. Jeffrey, Group cohomology construction of symplectic forms on certain moduli spaces, Internat. Math. Res. Notices 6 (1994), 245-249.

[10] L. Jeffrey, Symplectic forms on moduli spaces of flat connections on 2-manifolds, Proceeding of the Georgian International Topology Conference (Athen, GA, 1993), ed. par W. Kazez. AMS/IP Studies in Advanced Mathematics 2 (1997), 268-281.

[11] L. Jeffrey Group cohomology construction of the cohomology of moduli spaces of flat connections on 2 manifolds, Duke Math. J. 77 (1995), 407-429.

[12] F. C. Kirwan, Cohomology of quotients in symplectic and algebraic geometry, Princeton University Press, Princeton, N.J., 1984.

[13] K. Kodaira and D. C. Spencer, On deformations of complex analytic structures, I\& II, Ann. of Math. 67 No. 2 (1958), 328-466.

[14] S. Kumar and M. Vergne, Equivariant cohomology with generalised coefficients, Astérisque 215 (1993), 109-204.

[15] D. McDuff, The moment map for circle actions on symplectic manifolds, J. Geom. Phys. 5 (1988), 149-160. 
[16] M. Mumford and P. Newstead, Periods of moduli space of bundles on curves, Amer. J. Math. 90 (1968), 1200-1208.

[17] M. S. Narasimhan and C. S. Seshadri, Stable and unitary vector bundles on a compact Riemann surface, Ann. Math. 82 (1965), 540-567.

[18] S. Racanière, Cohomologie équivariante de $\mathbf{S U}(n)^{2 g}$ et application de Kirwan, Comptes Rendus Acad. Sc. 333 (2001), 103-108.

[19] A. Weitsman A Duistermaat-Heckman formula for symplectic circle actions, Math. Res. Notices 12 (1993), 309-312.

S. Racanière

Institut de Recherche Mathématique Avancée

Université Louis Pasteur et CNRS

Bureau 113

7 rue René Descartes

67084 Strasbourg Cedex

France

e-mail: racanier@irma.u-strasbg.fr

URL: http://www-irma.u-strasbg.fr/ racanier

(Received: May 28, 2002) 